\title{
Charged Domain Wall and Polar Vortex Topologies in a Room Temperature Magnetoelectric Multiferroic Thin Film
}

Kalani Moore

University of Limerick https://orcid.org/0000-0002-5962-2651

Eoghan O'Connell

University of Limerick https://orcid.org/0000-0001-5154-5308

Sinéad M. Griffin

Lawrence Berkeley National Laboratory

Clive Downing

Trinity College Dublin

Louise Colfer

Tyndall National Institute

Michael Schmidt

University College Cork

Valeria Nicolosi

Trinity College Dublin https://orcid.org/0000-0002-7637-4813

Ursel Bangert

University of Limerick https://orcid.org/0000-0002-7511-7663

Lynette Keeney ( $\square$ lynette.keeney@tyndall.ie)

Tyndall National Institute, University College Cork, 'Lee Maltings', Dyke Parade, Cork, Ireland.

https://orcid.org/0000-0003-0980-514X

Michele Conroy ( $\square$ michele.conroy@ul.ie)

University of Limerick https://orcid.org/0000-0002-6658-1819

Article

Keywords: Multiferroic, Polar, Domain Walls, Topologies, Vortex, thin film

Posted Date: September 8th, 2021

DOI: https://doi.org/10.21203/rs.3.rs-114541/v3

License: (c) (1) This work is licensed under a Creative Commons Attribution 4.0 International License.

Read Full License 
Version of Record: A version of this preprint was published at ACS Applied Materials \& Interfaces on January 19th, 2022. See the published version at https://doi.org/10.1021/acsami.1c17383. 


\section{Charged Domain Wall and Polar Vortex Topologies in}

\section{a Room Temperature Magnetoelectric Multiferroic}

\section{Thin Film}

Kalani Moore, ${ }^{1}$ Eoghan N. O'Connell, ${ }^{1}$ Sinéad M. Griffin,${ }^{2,3}$ Clive Downing, ${ }^{4}$ Louise Colfer, ${ }^{5}$ Michael Schmidt,${ }^{5}$ Valeria Nicolosi, ${ }^{4,6}$ Ursel Bangert, ${ }^{1}$ Lynette Keeney,${ }^{5 *}$ Michele Conroy ${ }^{1,7 *}$

1. Department of Physics, Bernal Institute, School of Natural Sciences, University of Limerick, Ireland

2. Materials Sciences Division, Lawrence Berkeley National Laboratory, CA, USA

3. Molecular Foundry, Lawrence Berkeley National Laboratory, CA, USA

4. Advanced Microscopy Laboratory \& AMBER, Trinity College Dublin, Ireland

5. Tyndall National Institute, University College Cork, Ireland

6. School of Chemistry, Trinity College Dublin, Dublin 2, Ireland

7. Department of Materials, Royal School of Mines, Imperial College London, UK

*Corresponding author emails: m.conroy@imperial.ac.uk and lynette.keeney@tyndall.ie

KEYWORDS: Multiferroic, Polar, Domain Walls, Topologies, Vortex, thin film 


\begin{abstract}
Multiferroic topologies are an emerging solution for future low-power magnetic nanoelectronics due to their combined tuneable functionality and mobility. Here, we show that in addition to being magnetoelectric multiferroic at room temperature, thin film Aurivillius phase $\mathrm{Bi}_{6} \mathrm{Ti}_{\mathrm{x}} \mathrm{Fe}_{\mathrm{y}} \mathrm{Mn}_{\mathrm{z}} \mathrm{O}_{18}$ is an ideal material platform for both domain wall and vortex topology based nanoelectronic devices. Utilising atomic resolution electron microscopy, we reveal the presence and structure of $180^{\circ}$ type charged head-to-head and tail-to-tail domain walls passing throughout the thin film. Theoretical calculations confirm the sub-unit cell cation site preference and charged domain wall energetics for $\mathrm{Bi}_{6} \mathrm{Ti}_{\mathrm{x}} \mathrm{Fe}_{\mathrm{y}} \mathrm{Mn}_{\mathrm{z}} \mathrm{O}_{18}$. Finally, we show that polar vortex type topologies also form at out-of-phase boundaries of stacking faults when internal strain and electrostatic energy gradients are altered. This study could pave the way for controlled polar vortex topology formation via strain engineering in other multiferroic thin films. Moreover, these results confirm the sub-unit-cell topological features play an important role in controlling the charge and spin state of Aurivillius phase films and other multiferroic heterostructures.
\end{abstract}




\section{INTRODUCTION}

Room temperature multiferroic materials, possessing coupled ferroelectric and ferromagnetic states, have exciting potential for use in future low-energy data-storage devices such as magnetoelectric-spin orbit logics for recurring neural networks. ${ }^{1-3}$ Layered oxide thin films such as the $\mathrm{Bi}_{2} \mathrm{O}_{2}\left(\mathrm{~A}_{\mathrm{m}-1} \mathrm{~B} \mathrm{~m}_{3 \mathrm{~m}+1}\right)$ Aurivillius phase offer a flexible template for designing new multiferroic materials, where $m$ is the number of perovskite units interleaved between the $\left(\mathrm{Bi}_{2} \mathrm{O}_{2}\right)^{2+}$ fluorite-type layers. ${ }^{4-5}$ Aurivillius phases are established ferroelectric materials with strong in-plane polarisations, ${ }^{6}$ high Curie temperatures $\left(>600^{\circ} \mathrm{C}\right)$ and fatigue-free energy storage performance..$^{7-9}$ The rare possibility of room temperature ferromagnetism within a ferroelectric framework is achieved in Aurivillius phases with the introduction of magnetic ions within the scaffold. ${ }^{10-14}{ }^{15} \mathrm{Bi}_{6} \mathrm{Ti}_{\mathrm{x}} \mathrm{Fe}_{\mathrm{y}} \mathrm{Mn}_{\mathrm{z}} \mathrm{O}_{18}$ ( $\mathrm{x}=2.80$ to 3.04; $\mathrm{Y}=1.32$ to $1.52 ; \mathrm{Z}=0.54$ to 0.64$)^{16}$ where $m=5$, is an example of such an ion-substituted multiferroic Aurivillius phase, displaying saturation magnetization $\left(\mathrm{M}_{\mathrm{S}}\right)$ values of $215 \mathrm{emu} / \mathrm{cm}^{3}$, with in-plane saturation polarization $\left(\mathrm{P}_{\mathrm{s}}\right)$ values of $>26 \mu \mathrm{C} / \mathrm{cm}^{2}$ and with experimentally proven magnetoelectric switching. $\mathrm{Bi}_{6} \mathrm{Ti}_{\mathrm{x}} \mathrm{Fe}_{\mathrm{y}} \mathrm{Mn}_{\mathrm{z}} \mathrm{O}_{18}$ (B6TFMO) can be thought of as a $2 \mathrm{D}$ nanostructured framework, with 5 perovskite cells sandwiched between dielectric $\left(\mathrm{Bi}_{2} \mathrm{O}_{2}\right)^{2+}$ layers. To maximise electrostatic interactions with the layer of oxygen anions in the $\left(\mathrm{Bi}_{2} \mathrm{O}_{2}\right)^{2+}$ layer, the more-highly-charged $\mathrm{Ti}^{4+}$ cations partition close to the $\left(\mathrm{Bi}_{2} \mathrm{O}_{2}\right)^{2+}$ layers. ${ }^{17}$ Lattice parameter differences between the unconstrained $\left(\mathrm{Bi}_{2} \mathrm{O}_{2}\right)^{2+}$ layer $(3.80 \AA)$ and that of the unconstrained perovskite block $(3.89 \AA)$ put the perovskite blocks closest to the $\left(\mathrm{Bi}_{2} \mathrm{O}_{2}\right)^{2+}$ layers under compressive stress. ${ }^{18-20}$ This provides elastic strain energy and drives the larger Mn cations towards the central perovskite layers in order to diminish interlayer stress. ${ }^{16}$ This magnetic cation partitioning increases the probability of nearest neighbor magnetic interactions in the central layer by up to $90 \%$ compared to a case where the magnetic cations are randomly distributed over the 5 available B-sites. This initiates true room temperature ferromagnetism, as opposed to the antiferromagnetic room temperature $\mathrm{BiFeO}_{3}{ }^{21}$ 
As the multiferroic-dielectric heterostructure is at the sub-unit cell scale, ${ }^{22-23}$ probing any changes within this heterostructure requires a characterisation technique with atomic scale spatial resolution. Aberration corrected scanning transmission electron microscopy (STEM) allows the multiferroic research community to investigate domain wall (DW) topologies at the sub-atomic scale. ${ }^{24-31}$ Using STEM we can quantify the atomic displacements and thus polarisation changes at and within topologies. Recently, Campanini, M., et al. ${ }^{32}$ in a STEM based paper, reported in-plane polarisation and lateral domains within $m=4 \mathrm{Bi}_{5} \mathrm{FeTi}_{3} \mathrm{O}_{15}$ Aurivillius thin films. No charged ferroelectric DWs were present and instead neutral ferroelectric DWs are located within the dielectric $\left(\mathrm{Bi}_{2} \mathrm{O}_{2}\right)^{2+}$ layers. In contrast, we confirm by STEM characterisation that charged $180^{\circ}$ head-head (H$\mathrm{H})$ and tail-tail (T-T) type DWs run through the entire film thickness of Mn containing B6TFMO thin film. This is the first study to experimentally report the atomic scale structure and composition of charged type DWs in a room temperature multiferroic Aurivillius phase material. Perfectly parallel H$\mathrm{H} / \mathrm{T}-\mathrm{T} 180^{\circ}$ walls of B6TFMO were calculated to be energetically unfavourable by density functional theory (DFT). These first principle calculations explain the observed angled orientation of the $180^{\circ}$ DWs during imaging across all of the samples. The DWs must bend in order to lower the energy cost while remaining in a charged state.

Additionally, we have found that polar vortex topologies were present in regions where out-ofphase boundaries (OPB) defects are spaced between 5 to 8 perovskite cells apart. In the regions where OPBs and associated stacking default defects were present, the magnetic $\mathrm{Mn}$ and Fe ion partitioning increased. Theoretical reports predict that while OPBs can suppress ferroelectricity, ${ }^{33}$ their presence results in elevated magnetic ion interactions, thereby increases extent of long-range magnetic order. ${ }^{12,}$ 34 Thus, inducing higher OPB densities could result in improved magnetoelectric coupling and device efficiency. Within this study, we investigate the role these OPB defects play in the formation of charged DWs and polar vortices. We observe increased partitioning of lower valence cations towards the vortex core, indicating that there is an increased electric conductivity at the ferroelectric vortex 
core. We map out the pico-meter scale atomic column shifts and unit cell deformation as demonstrated previously for other ferroic materials,${ }^{35-38}$ revealing the direct link between OPBs and polar vortices in Aurivillius phase thin films. In addition to data-storage capabilities, this work demonstrates the wider technological potential of B6TFMO, with prospects for application in energy-efficient nanoelectronics and DW devices.

\section{RESULTS \& DISCUSSION}

The $100 \mathrm{~nm}$-thick Aurivillius films in this study were synthesized by liquid injection chemical vapour deposition and the magnetoelectric multiferroic properties were confirmed in our previous reports. ${ }^{15,39}$ Figure 1 shows a model of the five-layered B6TFMO unit cell alongside an experimental confirmation of the atomic resolution STEM high-angle annular dark-field (HAADF) image of the structure. One ferroelectric block with one dielectric layer corresponds to half of one (0.5) Aurivillius phase unit cell. The Aurivillius phase materials are established ferroelectrics, strongly favouring in-

plane polarisation, with spontaneous electrical polarisation (Ps) of $\sim 50 \mu \mathrm{C} \mathrm{cm}^{-2},{ }^{40-41}$ as observed from previous macroscopic polarisation vs. electric field measurements, piezoresponse force microscopy measurements and $a b$ initio calculations. In this contribution, we use HAADF-STEM cross-sectional imaging to investigate polar behaviour within sections of the B6TFMO films. The large yellow arrows in Figure 1(a) and throughout this paper indicate the net polarisation vector for each ferroelectric layer. The polarisation vector was determined by the conventional approach of reverse B-site displacement mapping. ${ }^{32,42-43}$ The ferroelectric-dielectric hetero-structure within the B6TFMO unit cell leads to distinctive ferroelectric behaviour, where oppositely polarised perovskite cells co-exist side by side within the ferroelectric layer ${ }^{32}$ as demonstrated in Figure 1(b). The perovskite cell is pseudo-cubic with a ferroelectric dipole forming analogous to that in $\mathrm{BiFeO}_{3}$. The outer perovskite cells bonded to the dielectric layer are hyper-tetragonal and thus highly strained along the c-axis $\left(\varepsilon_{y y}\right)$ compared to $\mathrm{BiFeO}_{3}$, $\mathrm{BiTiO}_{3}$ or $\mathrm{PbTiO}_{3}$ unit cells. At these outer perovskite cells, the polarisation is larger and always point 
towards the dielectric layer. At the 3 perovskite cells in the centre of the layer, the $\varepsilon_{y y}$ strain relaxes and thus the polarisation state has a shallower potential well. ${ }^{44}$ In other words, with reduced $\varepsilon_{\mathrm{yy}}$ strain present, polarisation direction is largely determined by electrostatic stress and has a lower energy cost for switching.

(a)

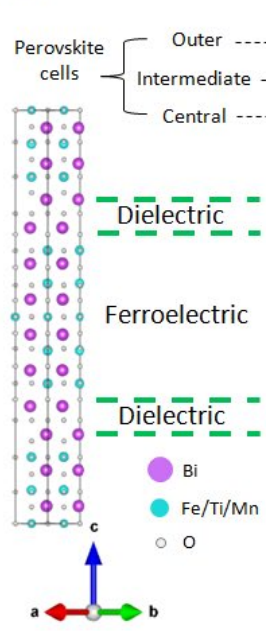

(d)

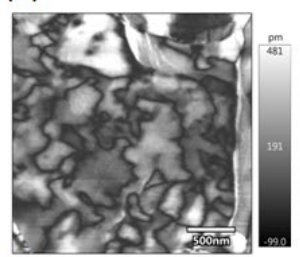

(e)

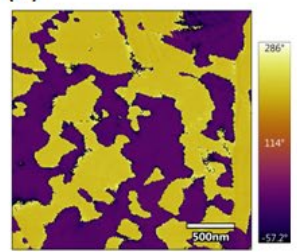

(b)

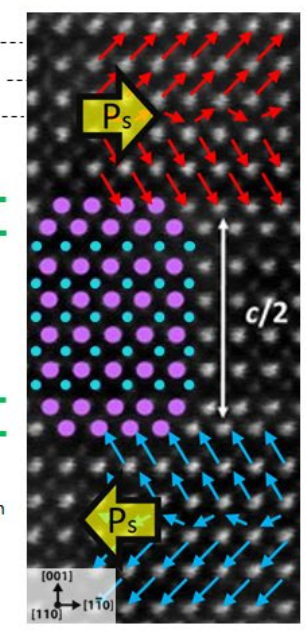

(f)

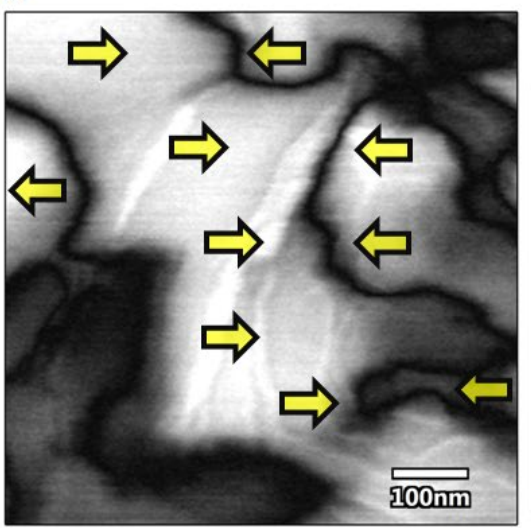

(c)

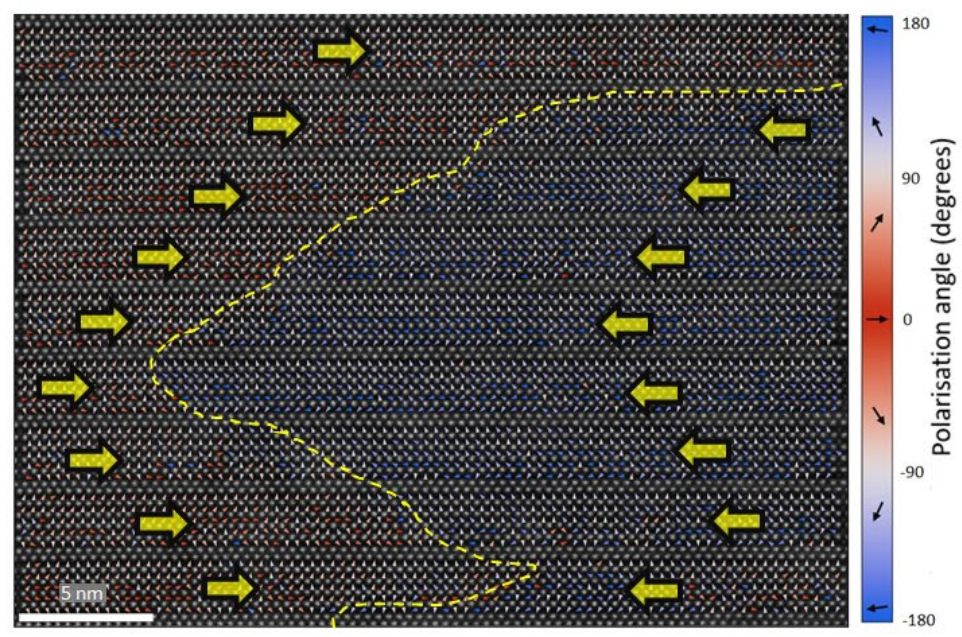

(g)

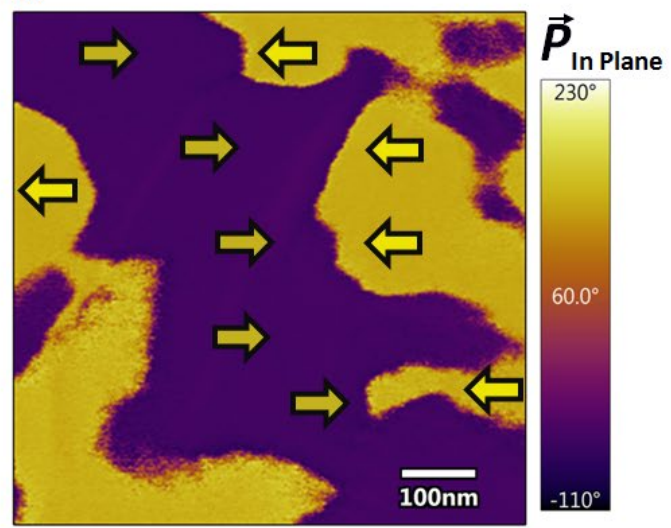

Figure 1. (a) Structural model of the five-layered Aurivillius phase B6TFMO unit cell (polar orthorhombic space group F2mm). One ferroelectric block with one dielectric layer corresponds to half of one Aurivillius phase unit cell. (b) The A-site and B-site atoms in the B6TFMO structure are overlaid on an atomic resolution high angle annular dark field image. Red/blue arrows are an illustration of the expected polarisation of the perovskite cells in the ferroelectric layer. The resulting in-plane net polarisation $\left(P_{s}\right)$ is indicated by the large yellow arrows. The ferroelectric (perovskite) and dielectric (fluorite) layers can clearly be distinguished by the Bi atom structures. (c) High angle annular dark field image overlaid with reverse B-site displacement polarisation map of each perovskite cell. Polarisation unit vectors are colour coded red/blue to illustrate the in-plane polarisation direction. The approximate position of a head-to-head $180^{\circ} \mathrm{DW}$ is marked by the dashed 
yellow line. Scalebar $=5 \mathrm{~nm}$. See Supplementary Figures $1 \& 2$ for maps of the polarisation averaged over $5 x$ 5 perovskite cells in the ferroelectric layer. In-plane piezoresponse force microscopy (PFM) (d) amplitude and (e) phase measurements of the sample surface demonstrate the magnitude of piezoresponse and polar orientation, respectively. A closer examination of PFM amplitude in $(f)$ and PFM phase in $(g)$ demonstrate that curved DWs separate oppositely orientated domains. The yellow arrows represent the direction of the in-plane polarization. Where these oppositely orientated polarisations have similar PFM amplitudes and meet $180^{\circ}$ either at head-to-head or tail-to-tail configurations, we anticipate that the DW will be charged at that region.

Figure 1(c) shows a vertical DW passing through the entire thin film in a curved fashion and through multiple Aurivillius phase unit cells. The blue/red colouring of the polarisation unit vectors indicates the in-plane vector component to the left/right respectively. Interestingly, net polarisation here is $\mathrm{H}-\mathrm{H}$ (head-to- head) oriented and rotates $180^{\circ}$ across the DW, thus is nominally charged in nature. $^{45}$ In-plane ferroelectric domains within cross-sections of 40 nm-thick $m=4 \mathrm{Bi}_{5} \mathrm{FeTi}_{3} \mathrm{O}_{15}$ Aurivillius films were previously observed in STEM experiments reported by Campanini, M., et al. ${ }^{32}$ Charged ferroelectric DWs were not observed however and instead neutral ferroelectric DWs are located within the dielectric $\left(\mathrm{Bi}_{2} \mathrm{O}_{2}\right)^{2+}$ layers, where the dipole experiences an interruption rather than a continuous rotation across perovskite cells. ${ }^{6,32}$ Nominally charged DWls were only observed for this $\mathrm{m}=4$ structure by surface piezoresponse force microscopy (PFM) measurements when the thickness was reduced to half a unit cell (approx $2 \mathrm{~nm}$ thick). ${ }^{6}$ The depth of the DW through the $100 \mathrm{~nm}$-thick film in our present work indicates that ferroelectric layers are coupled, and unlike previous reports of the even-layered $m=4 \mathrm{Bi}_{5} \mathrm{FeTi}_{3} \mathrm{O}_{15}$ phase, the ferroelectric layers are not fully electrostatically isolated by the dielectric layers in between. ${ }^{32}$

Lateral PFM imaging of our 100 nm-thick B6TFMO films confirms the polar nature of the films at the surface. Fig. 1 (d) to (e) demonstrates that the films are naturally self-polarized and a random mixture of domain states is exhibited, separated by $180^{\circ}$ degree DWs. As expected from the crystal symmetry of the $m=5$ Aurivillius phases, having an odd $m$ number of perovskite units, the polarization primarily lies along the lateral direction, with only minor polarization along the out-of- 
plane, vertical direction. A closer inspection of the domains and DW configurations in Fig. 1 (f) and (g) reveals the curved nature of the DWs at the surface, similar to that observed through the film in Fig 1 (c). We also observe regions where oppositely orientated in-plane domains have similar magnitudes of piezoresponse. Hence, when these oppositely orientated polarisations meet $180^{\circ}$ either at $\mathrm{H}-\mathrm{H}$ or $\mathrm{T}$ $\mathrm{T}$ (tail-to-tail) configurations along parts of the curved DW, we anticipate that the DW will be charged at that region.

H-H DWs have "bound positive charge" from the adjacent polarisations which should be screened by a negative surface charge density $\left(\sigma_{\mathrm{s}}\right)$ equal to $2 \times \mathrm{P}_{\mathrm{s} .}{ }^{45}$

In the case of B6TFMO where in-plane $\mathrm{P}_{\mathrm{s}}=0.5 \mathrm{C} / \mathrm{m}^{2},{ }^{40-41}$

$$
\sigma_{s}=2 \times 0.5 \mathrm{C} / \mathrm{m}^{2}=1 \mathrm{C} / \mathrm{m}^{2}
$$

To make sense of what this means on the atomic scale we convert it to units of $|\mathrm{e}| /$ perovskite cell. Where $|\mathrm{e}|=1.6 \times 10^{-19}$, or elementary charge, and perovskite cell is the average surface area of a perovskite cell, approximately $3.835 \AA$ x $4.5 \AA$. Then,

$$
\sigma_{\mathrm{S}}=\frac{(1)\left(3.835 \times 10^{-10}\right)\left(4.5 \times 10^{-10}\right)}{\left(1.6 \times 10^{-19}\right)}=1.09 \mid \mathrm{e} / / \text { perovskite cell }
$$

This calculated $\sigma_{\mathrm{s}}$ is of the same order as that found at strongly charged DWs in other perovskites. ${ }^{46}$ As charge is likely to be spread across the DW width for 5-8 perovskite cells, rather than be confined to a 2D surface, a more realistic number for charge density $(\rho)$ would be

$$
\rho=\frac{1.09}{6.5 \text { PK cells }}=0.17|\mathrm{e}| / \text { perovskite cell. }
$$

However, this value would vary depending on the local DW width. The screening charge could be provided by either $[\mathrm{Bi} / \mathrm{Ti} / \mathrm{Mn} / \mathrm{Fe}]$ vacancies or a local change in oxidation state. The existence of two-dimensional sheet of charge carriers passing through layers of a multiferroic film raises the possibility of electroresistance complementing magnetoresistance in a multiferroic tunnel junction memory device. ${ }^{47-48}$ 
Figure 2 displays the detail of the perovskite cell polarisation evolution across the $180^{\circ} \mathrm{H}-\mathrm{H}$ DW. The yellow traced domain wall position is approximated as running between the inflection point of the polarisation in the outer perovskite cells. The 5-8 perovskite cells of inhomogeneous net polarisation is similar in width to charged $\mathrm{DWs}$ in $\mathrm{BiFeO}_{3}$ but quite large for a ferroelectric domain wall. ${ }^{49}$ This infers a lower exchange energy allowed by a lower anisotropy cost. That is to say, the central perovskite cells seem to be more "polarisable" than structurally harder ferroelectrics with thinner DWs such as $\mathrm{PbTiO}_{3} .{ }^{50}$ The anisotropy cost of the DW is studied by examining rotation and $\varepsilon_{\mathrm{yy}}$ strain maps in Figure 2(b) and Figure 2(c), respectively. While no clear change in $\varepsilon_{\mathrm{yy}}$ strain was identified at the DW in Figure 2(c), the strong rotation of $\sim 6^{\circ}$ in Figure 2 (b) indicates a shearing response of the structure at the DW. The same effect is seen in $\mathrm{BiFeO}_{3} \mathrm{H}-\mathrm{T}$ DWs. ${ }^{49}$ The lack of strain difference between domains confirms that there is little anisotropy cost, allowing for a semi-continuous rotation of the polarisation i.e. Néel/Bloch-type behaviour. ${ }^{51}$ The property of central layers being "polarisable" is associated with negative capacitance, specifically in ferroelectric-dielectric multilayer heterostructures. ${ }^{52}$ However, in this case, the perovskite cell retains a larger $c / a$ ratio than pseudo-cubic bulk rhombohedral $\mathrm{BiFeO}_{3}$ so the effect is deemed unlikely. The demonstrated "structural softness" is consistent with a large magnetoelectric effect. ${ }^{53}$ Furthermore, the relatively low anisotropy associated with the domain wall indicates that switching is determined only by the electrostatic energy, with no elastic energy cost. These results imply that charged DWs in B6TFMO are likely to be highly mobile with low dielectric loss. 
(a)

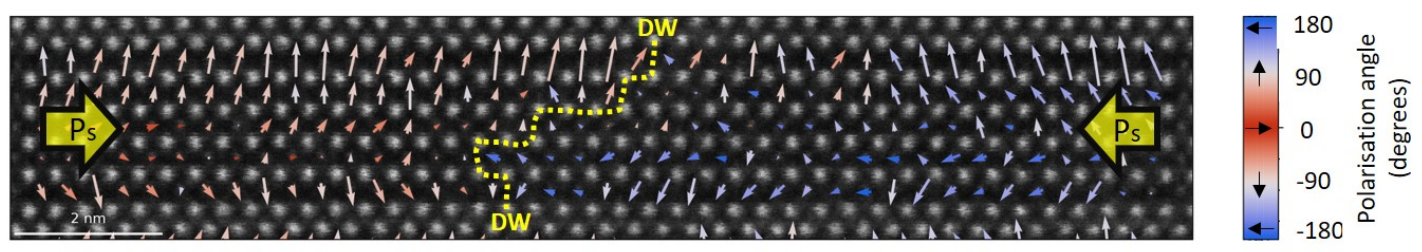

(b)

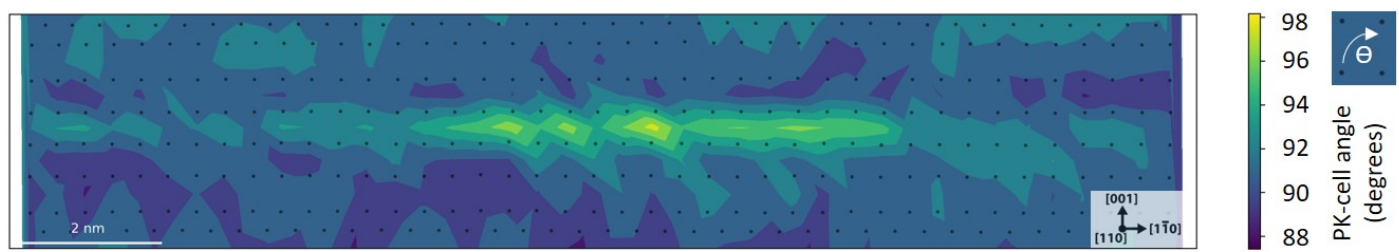

(c)

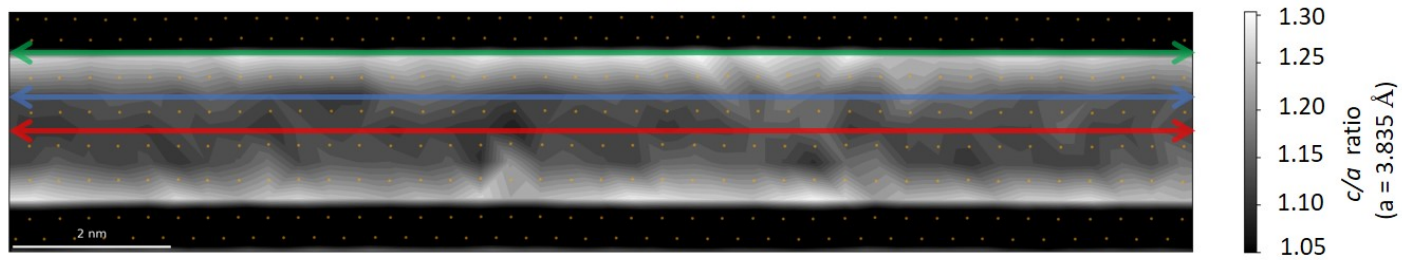

(d)

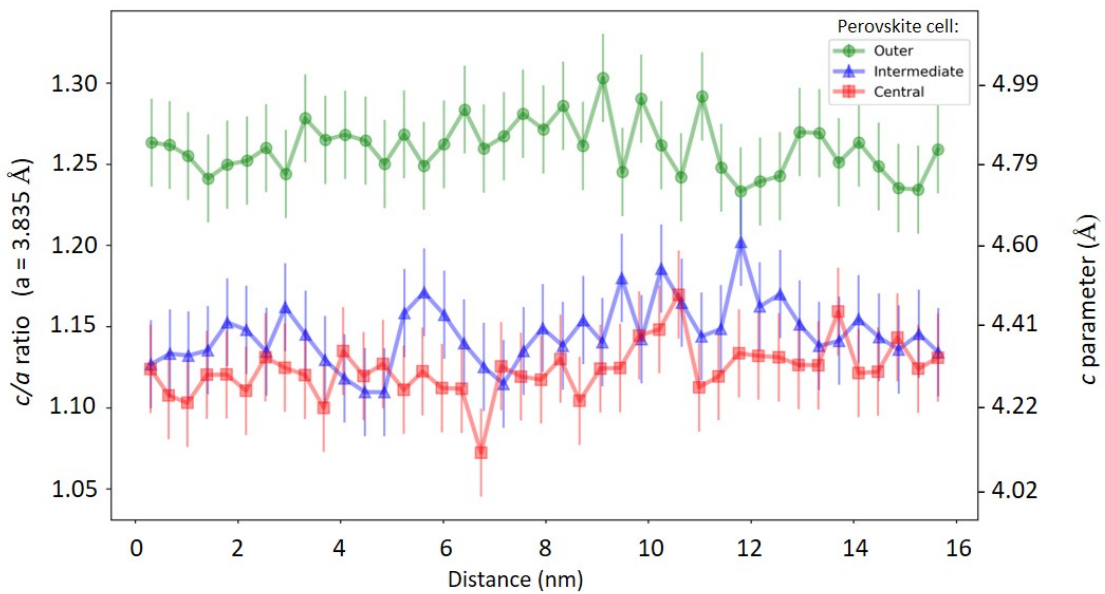

Figure 2: (a) Polarisation map across a head-to-head charged DW. The arrow lengths indicate the relative magnitude of the B-site displacement and hence the relative magnitude of the polarisation. A yellow dashed line indicates the approximate $D W$ position. The polarisation magnitudes are suppressed near the $D W$, indicating a width of approximately 5-8 perovskite cells. (b) Map of perovskite cell angles reveals $a \sim 6^{\circ}$ shearing at the $D W$. (c) map and (d) profiles of cla ratio comparing the outer, intermediate and central perovskite cells. The cla ratio is measured with respect to the average a parameter of 3.835 A, making cla equal to strain $\varepsilon_{y y}+1$. Error bars represent estimated measurement uncertainty. The measured a parameter varies $\pm 4 \%$ within the ferroelectric layer. This alters the actual measured cla values from those on the graph, but introduces more error while at the same time not changing the relationship between outer, intermediate and central perovskite cells. See Supplementary Figure 3 for complete results. Scalebars $=2 \mathrm{~nm}$. 
If one applies the knowledge gained from $\mathrm{BiFeO}_{3}$ research, then the lowest energy polarisation state is to point to one of the "corner" Bi atoms of the perovskite cell. This appears to contribute to the angled orientation of the DW, seen throughout the figures in this study. We performed first principles calculations to understand the cation site preference and DW energetics for B6TFMO domains. The full calculations details are given in the methods; here we summarize the main results.

Our unit cell comprised $\mathrm{Bi}_{24} \mathrm{Ti}_{11} \mathrm{Fe}_{6} \mathrm{Mn}_{3} \mathrm{O}_{72}$ which most closely matched the stoichiometry of the experimental crystal $\mathrm{B} 6 \mathrm{TFMO}-\mathrm{Bi}_{24} \mathrm{Ti}_{11.2} \mathrm{Fe}_{6.08} \mathrm{Mn}_{2.72} \mathrm{O}_{72}$-- while maintaining a feasible cell size for first-principles calculations. We fixed the 'inner' perovskite units to be Bi-Fe-O (green shaded polyhedra) and Bi-Mn-O (magenta shaded polyhedra), as indicated in Fig. 3, which is consistent with previous experimental and theoretical studies on cation ordering in Aurivillius phases. ${ }^{54}$ With this constraint, we explored the energetics of cation ordering for eight configurations of the remaining five cations as illustrated in Fig. 3. Our cation ordering energetics and resulting magnetic polarizations are also summarized in Fig. 3. Overall, we find the lowest-energy cation orders to have Mn ions in the 'inner' perovskite units - the lowest-energy configuration with Fe in the inner units (Figure 3 (d)) is over $800 \mathrm{meV}$ per unit cell higher in energy than the lowest-energy configuration with $\mathrm{Mn}$ in the inner units (Figure 3 (a)). These results are consistent with previous STEM experiments, ${ }^{16}$ which demonstrated a clear preference for Mn cations to partition into the central perovskite layer, but no significant preference for $\mathrm{Fe}$ to partition to the inner layers. Aside from the inner perovskite unit chemistry, we find that the preferred cation ordering is where the magnetic cations (Fe and $\mathrm{Mn})$ are separated, i.e. they share minimal common oxygen bonds. This general trend can be observed throughout the eight cation configurations considered - the lowest energy structures comprise of separated magnetic ( $\mathrm{Fe}$ and $\mathrm{Mn}$ ) cations, whereas the most unstable structures have clustered magnetic cations. For example, the highest-energy configuration, Fig. 3 (h), comprises of Fe and Mn atoms that are clustered together with the outer blocks comprising mostly of Ti-O polyhedral. The clustering of the $\mathrm{Fe}$ and $\mathrm{Mn}$ in the central layer results in more significant polyhedral tilting and stretching compared 
to when the magnetic cations are more spread out - this results in energy destabilizing tilting and polyhedral distortions that result in this higher-energy structure. Tilting and rotation of the octahedra are common features of the ferroelectric phase transitions in the Aurivillius phases and are energetically more favorable than octahedral deformations. All of our considered configurations have ferrimagnetic ordering with a net magnetic moments ranging from $1 \mu_{\mathrm{B}}$ to $40 \mu_{\mathrm{B}}$ per unit cell, depending on the particular cation ordering.

(a)

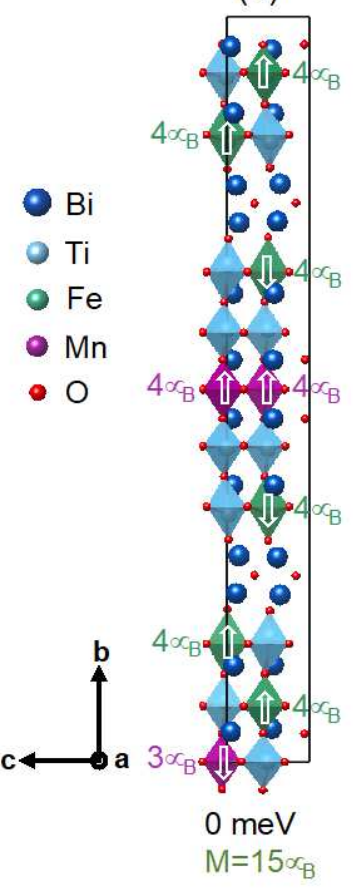

(b)

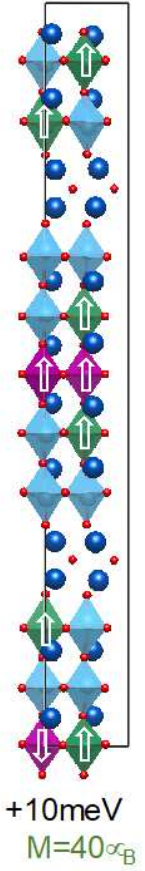

(c)

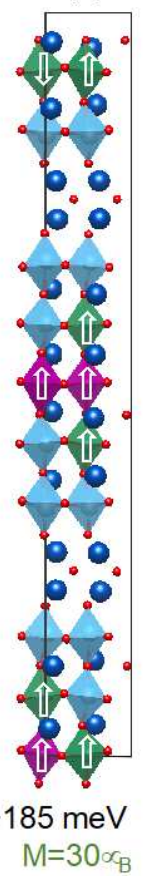

(d)

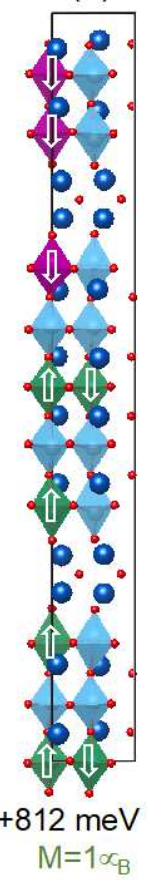

(e)

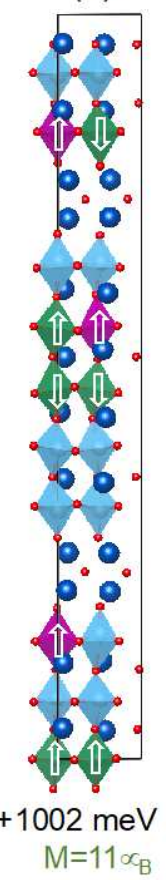

(f)

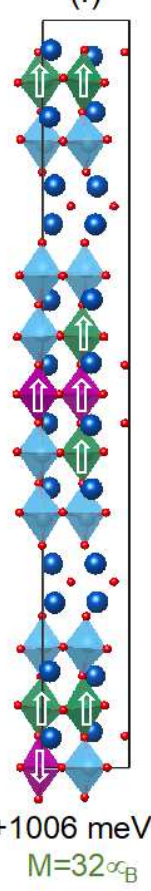

(g)

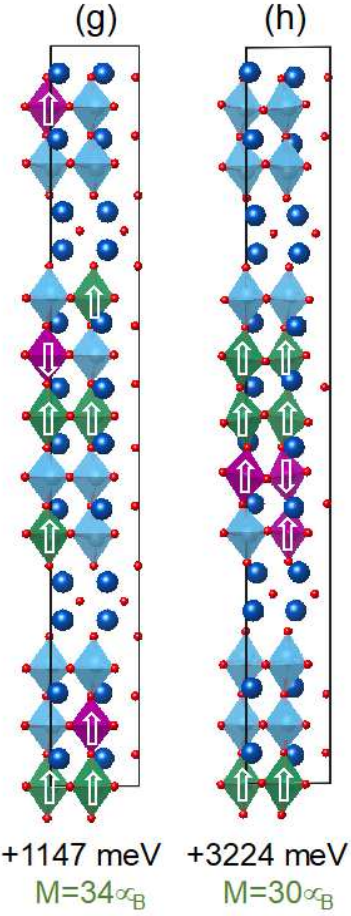

Figure 3 (a) Calculated Ti-Fe-Mn cation order structures using first-principles calculations for eight (a)-(h) different configurations. The crystal structures are the fully optimized (lattice parameter and internal coordinates) for the cation ordering scheme considered. The calculated relative energy of the various structures is given below each configuration relative to (a), which is set to $0 \mathrm{eV}$. The final calculated magnetic moment for the unit cell is also reported below each crystal structure. The final magnetic ordering for each structure is depicted with arrows on the Fe and Mn ions, with the local projected moments also shown for (a).

Finally, we calculate the domain wall energetics of two configurations - (i) H-H in plane (i.e. opposite electronic polarizations in c direction) and (ii) head-to-tail in the out-of-plane direction (i.e. opposite electronic polarizations in the b direction). We consider domains of double stoichiometry in the $\mathrm{c}$ direction, and fix the central atoms in each domain for each DW relaxation. We find the $\mathrm{H}-\mathrm{H}$ 
DW (i) to be very energetically unfavourable with a cost of $260 \mathrm{meV} /$ formula unit compared to the head-to-tail DW (ii). Therefore, we find that abrupt 90 degree DWs are highly unfavourable in this class of oxides. This is consistent with the experimental observations which have a staggered domain wall to avoid the highly unfavorable H-H DWs.

While in bulk $\mathrm{BiFeO}_{3}, c=a=3.96 \AA$, epitaxial strain $\left(\varepsilon_{\mathrm{xx}}\right)$ can result in rhombohedral and tetragonal phases with $c / a$ ratios up to 1.13 and 1.27 respectively. ${ }^{55}$ Fascinatingly, the measured $a$ spacing in B6TFMO (range: $3.68 \AA$ to $3.99 \AA$, uncertainty $+/-0.1 \AA$, average: $3.835 \AA$,) includes the $a$ spacing of $\mathrm{LaAlO}_{3}$ at $3.79 \AA$ on which the rhombohedral $(a>3.79 \AA)$ and tetragonal $(a<3.79 \AA)$ $\mathrm{BiFeO}_{3}$ phases can co-exist. ${ }^{56}$ This implies that individual perovskite cells in B6TFMO can exist flexibly in local rhombohedral or tetragonal phases. The natural $a$ spacing of the $\left(\mathrm{Bi}_{2} \mathrm{O}_{2}\right)^{2+}$ dielectric layer is calculated to be $3.80 \AA$. The average $a$ value agrees exactly with previous Aurivillius phase measurements, falling between the natural dielectric layer and perovskite cell $a$ values. ${ }^{18}$ Therefore the outer perovskite cells bonded to the dielectric layer are effectively under compressive epitaxial stress/strain $\left(-\varepsilon_{\mathrm{xx}}\right)$ for their $a$ parameter. Thus, in Figure $2(\mathrm{c})$, the observation of $c / a \approx 1.27$ in the outer perovskite cells can be explained as a transition to the hyper-tetragonal phase of the perovskite cell. Similarly, the observed $c / a \approx 1.10-1.15$ in the central and intermediate perovskite cells corresponds to a highly strained $\left(+\varepsilon_{\mathrm{yy}},-\varepsilon_{\mathrm{xx}}\right)$ rhombohedral perovskite cell. Therefore, a unit cell of B6TFMO can be considered as containing a morphotropic phase boundary between rhombohedral and tetragonal perovskite phases. 
(a)

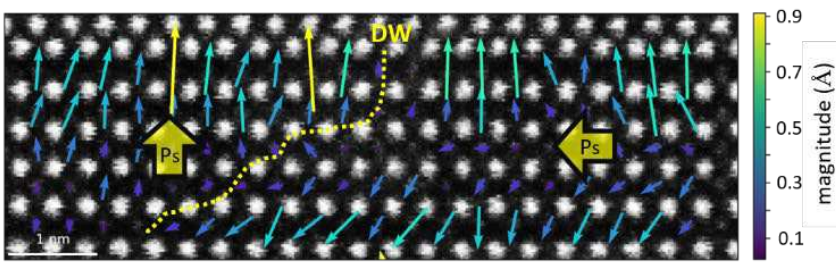

(b)

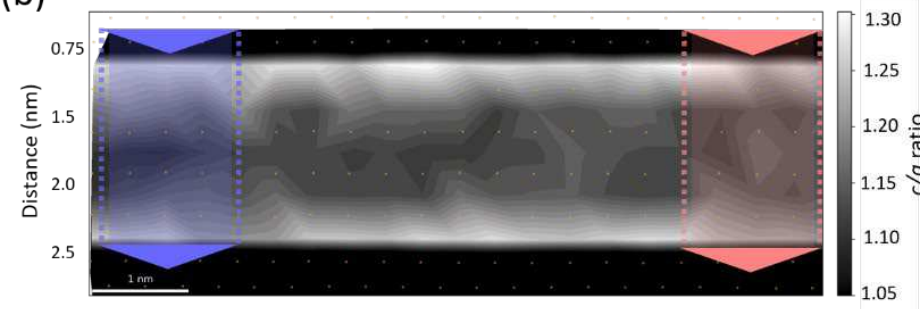

(c)

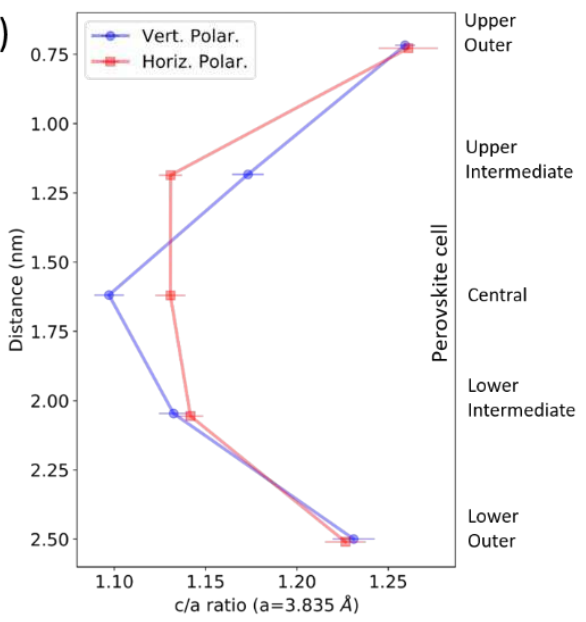

Figure 4: (a) Polarisation map of a head-to-tail DW. Vectors are coloured by magnitude of the B-site displacement. Polarisation magnitudes are suppressed in the lower perovskite cells and enhanced in the upper perovskite cells in the left domain. This results in a net vertical, c-axis polarisation as indicated by the large yellow arrow in the left domain. (b) c/a ratio map of (a) and (c) graph comparing line profiles across the caxis polarised "vertical" domain (blue) and the in-plane polarized "horizontal" domain (red) in (b). The shaded area between dotted lines marks the position of the profiles. The strain values in (c) are averaged over 5 perovskite cells, indicated by the arrow widths in (b). In (c) there is a clear asymmetry in the vertically polarized domain, demonstrating the link between strain and polarisation in the structure. Error bars represent standard error of the mean. Scalebars $=1 \mathrm{~nm}$.

In Figure 4(a) a second type of DW is analysed, where the net polarisation is head-to-tail (HT). There is a stark difference in configuration compared to the H-H DWs. Firstly, the net polarisation transition across the DW is sharp. Secondly, polarisation magnitudes are suppressed in the lower perovskite cells and enhanced in the upper perovskite cells in the left domain. This results in a net vertical, c-axis polarisation. H-T DWs are the most commonly observed type of DW in proper ferroelectrics. Usually in odd-layered Aurivillius phase materials, perfect mirroring along the c-axis is not possible due to symmetry and octahedral rotations. The sole contributor to out-of-plane polarisation is therefore expected to be the symmetry breaking central perovskite cell, with mirrored polarisation of the outer and intermediate perovskite cells. ${ }^{57-59}$ What we observe in the left domain of Figure 4(a) is completely different; polarisation is almost uniformly pointing up in every perovskite cell. The lower perovskite cells have a suppressed polarisation magnitude (B-site displacement) $\sim 0.1$ 
$\AA$, while the upper perovskite cells have an enhanced polarisation magnitude of $\sim 0.7 \AA$. For reference, the polarisation magnitude in bulk $\mathrm{BiFeO}_{3}$ is $\sim 0.2 \AA$. Faraz, A., et al. measured an out-of-plane ferroelectric response of $10 \mathrm{pm} / \mathrm{V}$ in these films. ${ }^{39}$

There is a clear correlation between this asymmetric c-axis polarisation and the $\varepsilon_{y y}$ strain of the ferroelectric layer in Figure 4(c). For the vertically polarised domain in Figure 4(a), the larger polarisation vectors for the upper-outer and upper-intermediate perovskite cells correspond to increased $\varepsilon_{y y}$ strain in Figure 4(c), compared with the in-plane polarised domain. While there is less $\varepsilon_{y y}$ strain in the central perovskite cell compared with the in-plane domain, there is no $\varepsilon_{y y}$ strain difference between vertical and in-plane domains for the intermediate and outer perovskite cells at the bottom of the layer, where the polarisation is suppressed. For the horizontally polarised domain in Figure 4 , both $\varepsilon_{y y}$ strain and polarisation vectors are symmetrical. The difference in strain states between the two domain types means there is a relatively high anisotropy cost for the DW. This manifests itself in the sharp, well-defined, H-T transition across 1-2 perovskite cells, contrasting with the wider H-H DW in Figure 2, but still following the same angled orientation. From EELS mapping (Supplementary Figure 4) there is a clear indication of oxygen depletion at the DW. Oxygen vacancies are common in perovskites, and normally found even at H-T DWs purely due to the strain field present, without needing to provide electrostatic screening. ${ }^{60-61}$

A further illustration of the interplay between strain and polarisation is shown in Figure 5, where ferroelectric polar vortices form. The rotating polarisation of the vortices are mapped out in Figure 5 (c) and (d). The vortex occurs as a form of $180^{\circ}$ polarisation transition between T-T domains. As such, the vortex must be charged to the same extent as the H-H DW in Figure 2, with average $\boldsymbol{\sigma}_{\mathbf{s}}=$ $1.09 e$ per perovskite cell. Although the DW width is similar to the $\mathrm{H}-\mathrm{H}$ case in Figure 2, the defined vortex polarisation suggests the charge density is concentrated at the vortex core,${ }^{62}$ further increasing the relative conductivity difference versus the domain. 

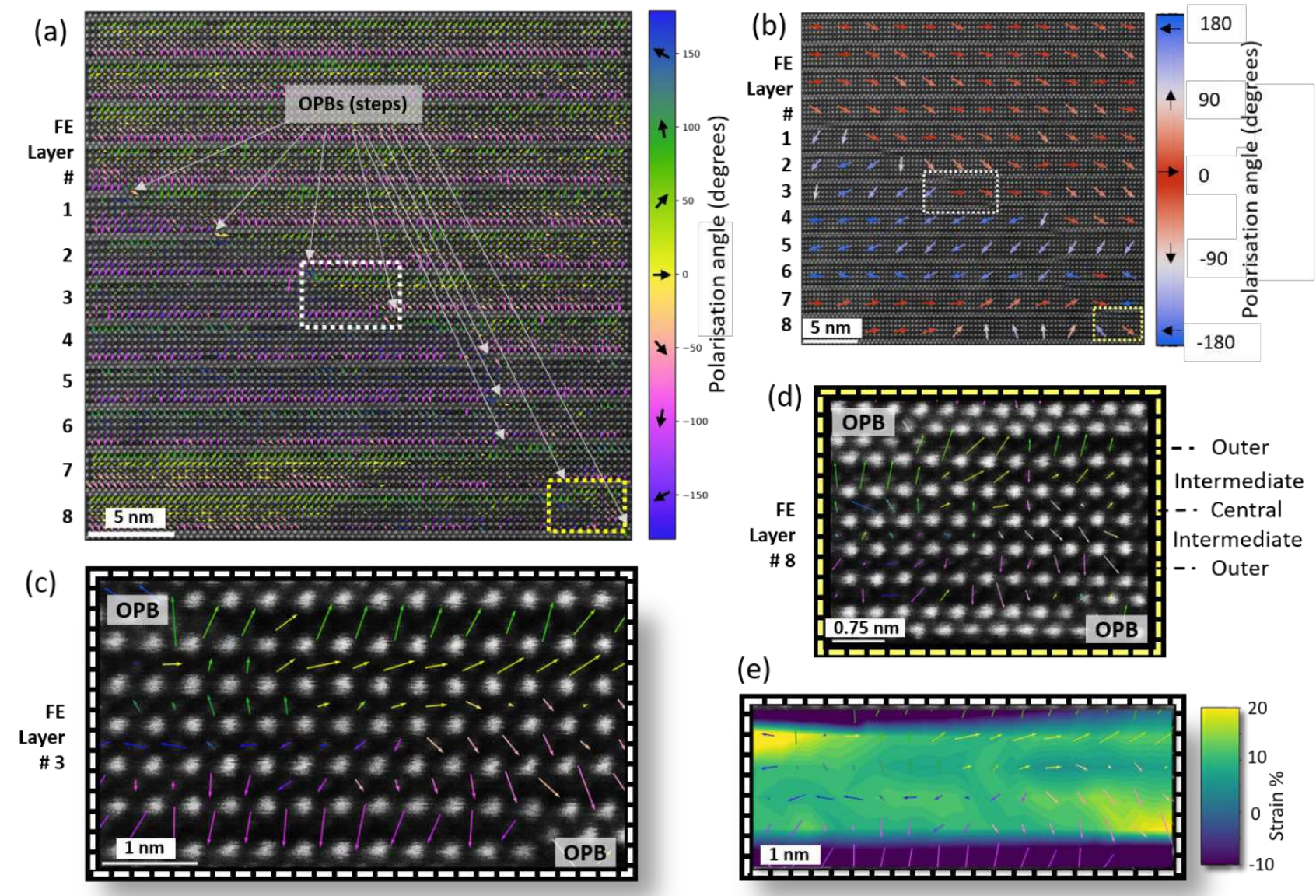

(e)

Figure 5 (a) Polarisation map of a large area containing DWs pinned at OPBs. The polarisation vector colours represent their angle relative to the $x$-axis. Two vortices are present, marked by dashed boxes. (b) The same map as (a), with polarisation vectors summed over $5 \times 5$ perovskite cells and colour coded red/blue to demonstrate the T-T character of the DWs. (c) Enlarged area from the white dashed box in (a) detailing the polarisation vortex which forms where T-T DWs occur in the vicinity of OPBs. (d) Enlarged area from the yellow dashed box in (a) where another vortex has formed. Perovskite cells are labelled to demonstrate the change in structure at the OPB. (e) The polarisation vectors from (b) overlaid on a $\varepsilon_{y y}$ strain map of the same area. $\varepsilon_{y y}$ strain is defined at the change of the c parameter from the average a parameter ( $3.835 \AA$ ), with $0 \%$ representing a pseudo-cubic structure. (c) and (d) share the same polarisation colour bar as (a). See Supplementary Figures S5-7 for more details.

Sub-unit cell defects, such as out-of-phase boundary (OPB) defects are a common occurrence in materials of high structural anisotropy, such as the Aurivillius phases, and are characterized by displacement of a fraction of a lattice parameter $(\mathrm{c} / \mathrm{x})$ between two neighbouring regions parallel to the c-direction. ${ }^{63}$ They appear as a "step" in the dielectric and ferroelectric layer and are energetically 
preferred to dislocations for relieving out-of-plane stress in the film. Disruptions of the Aurivillius phase lattice by OPBs have a marked influence on the internal elastic strain and electrostatic energy gradients within the structure and further drive larger cations away from the OPB defect towards the central layers of the structure. ${ }^{16}$ From Figure $5(\mathrm{a})$ it is evident that the line of OPB defects propagates diagonally down through the film, similar to the preferred orientation of the H-H DW in Figure 2. Figure 5(b), in which the polarisation vectors are summed over $5 \mathrm{X} 5$ perovskite cells, shows that the T-T DW broadly follows the OPBs down through the layers of the film. The T-T DW decouples from the OPB defects in ferroelectric layers 5-7, which instead contain a $180^{\circ}$ neutral DW and a $180^{\circ} \mathrm{H}-\mathrm{H}$ DW. Ferroelectric layer 8 contains both a H-H DW and a T-T vortex. This is shown in detail in Figure $5(d)$. The rich variety of features within just $20 \mathrm{~nm}$ is testament to the complex electrostatic screening in the film. As shown in the low magnification images of supplementary Figure S8, OPB defects are found throughout different samples grown during different growth runs.

Vortices are more traditionally associated with ferromagnetic systems, where there is no anisotropy cost to extend the transition of the magnetisation direction, thus lowering the exchange energy at the DW. Ferroelectric vortices have previously been identified in thin films of $\mathrm{BiFeO}_{3}$ and $\mathrm{PbTiO}_{3}$ multilayers, ${ }^{64-70}$ but remain a novel occurrence. However the coupling of multiferroic order parameters is known to induce a chirality at DWs, ${ }^{71-72}$ thus as this material is multiferroic and coupled to strain at the OPBs it is in fact not surprising that vortices can form. To examine the strain state of the vortex we compare the c-axis strain map $\left(\varepsilon_{\mathrm{yy}}\right)$ in Figure 5(d) to the perovskite cell polarisation in Figure 5(c). There is an asymmetric $\varepsilon_{y y}$ strain gradient either side of the vortex, but no strain change at its centre. Thus the anisotropy cost, DW width and the exchange energy (determined by the curl of the polarisation) should show the same behaviour as the H-H DW in Figure 2. We reason that the vortex occurs due to geometric/strain effects controlling the polarisation as demonstrated in Figure 5(d). Increased $\varepsilon_{\mathrm{yy}}$ strain to the upper-left and lower-right of the vortex core corresponds to OPBs adding an extra perovskite cell polarised up and down respectively on either side, i.e. the increased $\varepsilon_{y y}$ 
strain is caused by the "steps". Furthermore, the central perovskite cells, which provide the in-plane polarisation are displaced.

In Figure 5(c) and (d), when the lower central perovskite cell on the left becomes the intermediate perovskite cell after the OPB, the polarisation turns downward. When the upper central perovskite cell on the right becomes the intermediate perovskite cell after the OPB, the polarisation turns upward. Thus, the $180^{\circ} \mathrm{T}-\mathrm{T}$ DW, instead of behaving similar to the H-H DW in Figure 2, becomes a vortex with a well-defined polar rotation. In line with this explanation, we observe that the lateral distance between successive OPBs must be similar to the $180^{\circ}$ charged DW width, 5-8 perovskite cells, for the vortex to form. The factors affecting vortex formation in each of the ferroelectric layers \#1-8 in Figure 5(a) are detailed in Table 1.

\begin{tabular}{|l|l|l|l|}
\hline Ferroelectric Layer & $\begin{array}{l}\text { Charged DW present } \\
\text { between OPBs? }\end{array}$ & $\begin{array}{l}\text { OPBs spaced 5-8 } \\
\text { perovskite cells apart? }\end{array}$ & Vortex present? \\
\hline 1 & Yes & No & No \\
\hline 2 & Yes & No & No \\
\hline 3 & Yes & Yes & Yes \\
\hline 4 & Yes & No & No \\
\hline 5 & No & No & No \\
\hline 6 & No & No & No \\
\hline 7 & No & Yes & No \\
\hline 8 & Yes & Yes & Yes \\
\hline
\end{tabular}

Table 1: Comparison of the factors affecting vortex formation in the ferroelectric layers containing OPBs presented in Figure $5(a, b)$.

Moving from the perovskite cell to the ferroelectric layer scale, there is evidence of polarisation coupling through multiple ferroelectric layers in each of the multilayer maps. The dielectric layers do not fully isolate the ferroelectric layers. In the film, the polarisation can rotate freely towards the viewing axis, adding an extra degree of freedom to the system. PFM results in Fig. 1 (d) to (g) and from other Aurivillius phase materials show that nominally charged DWs are evident in as-grown films, ${ }^{6}$ so the ferroelectric results from STEM HAADF presented here appear to be representative. Of 
course as always one should consider whether ferroelectric behaviour may be altered in a cross-section STEM lamella compared to the as-grown thin film. Thin lamellae strongly favour polarisation within the plane of the lamella to reduce surface charging. The conduction mechanisms in similar Aurivillius phase films vary depending on the strength of the applied field but both ionic conductivity and electron hopping conductivity have an important contribution according to Song, D., et al ${ }^{73}$

These results offer a detailed insight into the ferroelectric behaviour of ion-substituted Aurivillius phase thin films. However, at the nanoscale, there remains significant questions to be answered. For instance, the structure of Aurivillius phase materials offers intriguing hints pointing towards localised charge separation. Each ferroelectric layer has a sharp transition from up to down polarisation across the 5 perovskite cells and thus one could consider this transition as a horizontal DW in the ferroelectric layer. The central perovskite cell should then contain positive screening charge, with the dielectric layer containing negative screening charge. ${ }^{17}$

In our previous work, ${ }^{16}$ we experimentally identified that Mn has a preference for concentrating towards the central perovskite cell and thus plays a key role generating room temperature ferromagnetism. Similar atomic scale STEM energy dispersive X-ray (EDX) spectroscopy mapping for this study was performed in the same OPB defect region as in Figure 4 (c) and is presented in Supplementary Figure S9. We observe an augmented partitioning of cations with a decreased positive charge (Mn with overall nominal valence of $3+$ ) away from the OPB defect regions and there is a promoted concentration of lower valence cations at the central perovskite layers. Conversely, there is a promoted partitioning of cations with increased positive charge (Ti with presumed overall nominal valence of $4+$ ) towards the defect regions and the outer perovskite layers. This indicates OPB defects provoke electrostatic energy changes and that there is an increased electric conductivity at the ferroelectric vortex core, similar to electric field gradient observations for $\mathrm{PbTiO}_{3} / \mathrm{SrTiO}_{3}$ multilayers ${ }^{62}$ and $\mathrm{BiFeO}_{3}{ }^{74} \mathrm{Mn}^{3+}$, the nominal oxidation state in B6TFMO to achieve charge balance, is known to disproportionate into $\mathrm{Mn}^{2+}$ and $\mathrm{Mn}^{4+} \cdot{ }^{75}$ The transition to $\mathrm{Mn}^{2+}$ and $\mathrm{Mn}^{4+}$ provides a likely source of 
electrons and holes for charge screening. Although outside the scope of this paper, charge separation between the centre and edges of each ferroelectric layer is thus a potentially interesting phenomenon to be further investigated by atomic scale fine structure EELS.

\section{CONCLUSION}

In summary, we have demonstrated the formation of both H-H and T-T DW type topologies within multiferroic B6TFMO thin films by cross section STEM and top surface PFM experimental observations. The DWs are coupled between ferroelectric layers and are not altogether electrostatically isolated by the dielectric layers in between. The DW topologies are highly charged with average $\sigma \_s$ $=1.09|\mathrm{e}|$ per perovskite-cell and $\rho \sim 0.17|\mathrm{e}|$ per perovskite-cell. The exact nature of screening charge is yet to be determined but local charge separation is likely due to the intrinsically opposing polarisation within the ferroelectric layer. H-T domains exhibit c-axis polarisation and the accompanying strain asymmetry signals electromechanical coupling. It is important to reemphasise that the multiferroic Aurivillius phase B6TFMO contains a naturally occurring dielectric-ferroelectric heterostructure and a morphotropic phase boundary, meaning distinctive ferroelectric behaviour is intrinsic.

Secondly, we show that this material system is a rich landscape to explore non-traditional ferroelectric DWs such as chiral topologies and thus potential spin to charge coupling at such topologies. We show that OPB boundary defects induced complete 2D polar vortices, if separated by a distance similar to the DW width. The atomic scale characterisation of the vortices gives insight into the scale and magnitude at which locally induced elastic strain and electrostatic energy changes can influence polarisation chirality. We hope this study relating to anisotropy and exchange energy at different DWs, can inform and spur increased interest into the magnetoelectric coupling at chiral topologies formed in Aurivillius phases and other multiferroic thin films. These results highlight the 
B6TFMO Aurivillius phase as an ideal platform for multiferroic topology engineering, and thus lowpower electric-field controlled magnetic device applications.

\section{METHODS}

\section{Crystal Growth:}

B6TFMO films were synthesized on c-sapphire substrates by liquid injection chemical vapour deposition (LI-CVD) methods ${ }^{39}$ and post-annealed at $850^{\circ} \mathrm{C}(1123 \mathrm{~K})$. The average stoichiometry, as determined by HR-SEM (high resolution scanning electron microscopy) with EDX, was $\mathrm{Bi}_{6} \mathrm{Ti}_{3.04} \mathrm{Fe}_{1.42} \mathrm{Mn}_{0.54} \mathrm{O}_{18}$.

\section{Electron Microscopy:}

Cross-sections of the B6TFMO films were prepared using a Thermo Fisher Scientific Dual Beam Helios NanoLab 600i and G4 model focused ion beam (FIB) and were mounted on a Cu based TEM grid. The sample was thinned via the FIB Ga beam first at $30 \mathrm{kV}$ and $93 \mathrm{pA}$, then $5 \mathrm{kV}$ and $43 \mathrm{pA}$ and then $2 \mathrm{kV}$ and $16 \mathrm{pA}$. The samples were then further thinned and polished to sub $30 \mathrm{~nm}$ using a Fischione $1020 \mathrm{Ar}$ ion based plasma cleaner prior to STEM imaging and EDX/EELS analysis. Energy filtered images acquired at $300 \mathrm{kV}$ on an Thermo Fisher Scientific Titan TEM with Gatan Tridiem Energy Filtering system demonstrated that thicknesses of the regions used for imaging were $<30 \mathrm{~nm}$. Imaging and analysis was performed on a NION UltraSTEM 200 operating at $200 \mathrm{kV}$ and a Thermo Fisher Scientific Titan Themis operating at 300kV. A Gatan Enfinium and GMS 2.0 was used for EELS acquisition and analysis.

Atom position finding and 2D Gaussian refinement were completed with the Atomap Python package. ${ }^{76}$ Image analysis and mapping, as well as polarisation vector analysis, were completed using the TEMUL Toolkit Python package. ${ }^{77}$ Supplementary material strain analysis was carried out by geometric phase analysis using Stem Cell. ${ }^{78}$ 


\section{Piezoresponse Force Microscopy:}

Electromechanical responses of the films were measured by PFM using an Asylum Research MFP$3 \mathrm{D}^{\mathrm{TM}}$ AFM in contact mode equipped with a HVA220 Amplifier for PFM and a cantilever scan angle of $90^{\circ}$. The Dual AC (alternating current) Resonance Tracking Piezoresponse Force Microscopy (DART-PFM) mode was used to boost both the vertical and lateral piezo signals. In this mode, the PFM signal is measured at the tip-sample contact resonance frequency, with higher signal-to-noise ratio compared with other frequencies. Topographical cross-talk is reduced using an amplitude feedback loop which tracks the contact resonance frequency so that drive frequencies are adjusted accordingly as the probe scans over the changing sample topography. Application of an $\mathrm{AC}$ bias to a conductive tip during contact mode imaging $\left(\mathrm{V}_{\text {tip }}=\mathrm{V}_{\mathrm{AC}} \cos (\omega \mathrm{t})\right)$ results in surface displacement $(d)$ and deflection of cantilever due to the converse piezoelectric effect, with both normal and in-plane components. Olympus AC240TM Electrilevers, Ti/Pt coated silicon probes (Al reflex coated, $15 \mathrm{~nm}$ tip radius, $70 \mathrm{kHz}$ resonant frequency) were used for PFM and topography imaging. The angular torsion of the cantilever as it oscillates was monitored in lateral PFM measurements. The drive frequencies were operated near contact resonance for lateral $(670-720 \mathrm{kHz})$ and vertical $(250-280 \mathrm{kHz}) \operatorname{modes}$ respectively with a probing signal of $1.0 \mathrm{~V}_{\mathrm{AC}}$.

\section{Density functional theory calculations:}

Density functional theory (DFT) calculations were carried out on 140-atom unit cells for the cation ordering calculations and 280 -atom unit cells for the DW energetics calculations of $\mathrm{Bi}_{24} \mathrm{Ti}_{11} \mathrm{Fe}_{6} \mathrm{Mn}_{3} \mathrm{O}_{72}$. Our calculations used the Vienna Ab initio Simulation Package (VASP) ${ }^{79-80}$ with projector augmented wave (PAW) pseudopotentials ${ }^{81}$ and Perdew-Burke-Ernzerhof exchange correlational functionals. ${ }^{82}$ We treat the $\mathrm{Bi}(6 s, 6 p)$, $\mathrm{Ti}(3 d, 4 s), \mathrm{Fe}(3 d, 4 s), \mathrm{Mn}(3 d, 4 s)$ and $\mathrm{O}(2 s, 2 p)$ as valence. We used a planewave cut-off energy of $600 \mathrm{eV}$ and a Monkhorst-Pack k-point grid of 4x1x4 for the 140 -atom unit cell and $2 \times 1 \times 4$ for the 280 -atom unit cells. To correct for the known underlocalization of the $d$ - 
orbitals of $\mathrm{Mn}$ and Fe, we applied a Hubbard-U correction of $3 \mathrm{eV}$ and $4 \mathrm{eV}$ respectively, consistent with previous works on transition-metal oxides, ${ }^{83}$ with the rotationally invariant version of GGA+U by Dudarev et al. ${ }^{84}$ We performed spin-polarized calculations to optimize the lattice parameters and internal coordinates until the forces on each atom were less than $0.01 \mathrm{eV} \AA^{-1}$. For all of our cation ordering configurations, we calculated the ferromagnetic (FM) order, and three different antiferromagnetic (AFM) orders, with the final magnetic order relaxing to a ferrimagnetic case for each, as depicted in Fig. 3.

\section{Author Contributions}

M.C. and L.K. conceived and led the project. L.K. developed the LI-CVD processes, L.K. and L.C. synthesized the B6TFMO thin films, L.K. performed PFM measurements. M.S. and M.C. produced the FIB samples. C.D. and M.C. performed the STEM imaging. C.D. performed the STEM EDX and EELS, while V.N. supervised this work. K.M., E.O'C. and L.C. performed the STEM data processing. S. M. G. performed the ab initio calculations. K.M. and M.C. lead the manuscript writing with input from all the co-authors.

\section{ACKNOWLEDGMENTS:}

This research was supported by the US-Ireland R\&D Partnership Programme (grant no. USI 120) and the Science Foundation Ireland (16/US/3344). The work at the Molecular Foundry was supported by the Office of Science, Office of Basic Energy Sciences, of the U.S. Department of Energy. M.C. acknowledges funding from Science Foundation Ireland (SFI) Industry Fellowship (18/IF/6282) and Royal Society Tata University Research Fellowship (URF $\backslash R 1 \backslash 201318)$. L. K. acknowledges funding from the Royal Society and SFI University Research Fellowships UF 140263

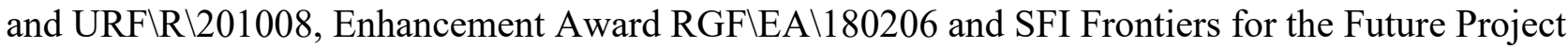

19/FFP/6475. S. M. G. acknowledges support by the Microelectronics Co-Design Research 
Program, under the Office of Science of the U.S. Department of Energy under Contract No. DEAC02-05CH11231. This research used resources of the National Energy Research Scientific Computing Center (NERSC), a U.S. Department of Energy Office of Science User Facility located at Lawrence Berkeley National Laboratory, operated under the same contract no.

\section{Ethics Declaration:}

The authors declare no competing financial or non-financial interests.

\section{REFERENCES}

1. Spaldin, N. A.; Ramesh, R., Advances in magnetoelectric multiferroics. Nature Materials 2019, 18 (3), 203-212.

2. Manipatruni, S.; Nikonov, D. E.; Young, I. A., Beyond CMOS computing with spin and polarization. Nature Physics 2018, 14 (4), 338-343.

3. Gradauskaite, E.; Meisenheimer, P.; Müller, M.; Heron, J.; Trassin, M., Multiferroic heterostructures for spintronics. Physical Sciences Reviews 2021, 6 (2).

4. $\quad$ Keeney, L.; Schmidt, M.; Amann, A.; Maity, T.; Deepak, N.; Faraz, A.; Petkov, N.; Roy, S.; Pemble, M. E.; Whatmore, R. W. In Novel approaches for genuine single phase room temperature magnetoelectric multiferroics, Nanoscale Ferroelectrics and Multiferroics: Key processing and characterization issues, and nanoscale effects, Wiley: 2016; pp 789-829.

5. Hervoches, C. H.; Snedden, A.; Riggs, R.; Kilcoyne, S. H.; Manuel, P.; Lightfoot, P., Structural behavior of the four-layer Aurivillius-phase ferroelectrics SrBi4Ti4O15 and Bi5Ti3FeO15. J. Solid State Chem. 2002, 164, 280.

6. Gradauskaite, E.; Campanini, M.; Biswas, B.; Schneider, C. W.; Fiebig, M.; Rossell, M. D.; Trassin, M., Robust In-Plane Ferroelectricity in Ultrathin Epitaxial Aurivillius Films. Advanced Materials Interfaces 2020, 7 (14), 2000202.

7. de Araujo, C. A. P.; Cuchiaro, J. D.; McMillan, L. D.; Scott, M. C.; Scott, J. F.; Mc Millan, D. L.; Scott, M. C.; Scott, J. F.; McMillan, L. D.; Scott, M. C., Fatigue-Free Ferroelectric Capacitors with Platinum Electrodes. Nature 1995, 374 (6523), 627.

8. Park, B. H.; Kang, B. S.; Bu, S. D.; Noh, T. W.; Lee, J.; Jo, W., Lanthanum-Substituted Bismuth Titanate for Use in Non-Volatile Memories. Nature 1999, 401 (6754), 682.

9. Pan, Z.; Wang, P.; Hou, X.; Yao, L.; Zhang, G.; Wang, J.; Liu, J.; Shen, M.; Zhang, Y.; Jiang, S., Fatigue-Free Aurivillius Phase Ferroelectric Thin Films with Ultrahigh Energy Storage Performance. Advanced Energy Materials 2020, 10 (31), 2001536.

10. Yang, B.; Song, D.; Wei, R.; Tang, X.; Hu, L.; Yang, J.; Song, W.; Dai, J.; Zhu, X.; Sun, Y., $\mathrm{Ni}$ doping dependent dielectric, leakage, ferroelectric and magnetic properties in Bi7Fe3xNixTi3O21 thin films. Applied Surface Science 2018, 440, 484-490. 
11. Zhai, X.; Grutter, A. J.; Yun, Y.; Cui, Z.; Lu, Y., Weak magnetism of Aurivillius-type multiferroic thin films probed by polarized neutron reflectivity. Physical Review Materials 2018, 2 (4), 044405.

12. Birenbaum, A. Y.; Ederer, C., Potentially Multiferroic Aurivillius Phase Bi5FeTi3 O15: Cation Site Preference, Electric Polarization, and Magnetic Coupling from First Principles. Phys. Rev. B: Condens. Matter Mater. Phys. 2014, 90 (21), 214109.

13. Algueró, M.; Pérez-Cerdán, M.; del Real, R. P.; Ricote, J.; Castro, A., Novel Aurivillius Bi4Ti3-2xNbxFexO12 phases with increasing magnetic-cation fraction until percolation: a novel approach for room temperature multiferroism. Journal of Materials Chemistry C 2020, 8 (36), 12457-12469.

14. Koval, V.; Skorvanek, I.; Viola, G.; Zhang, M.; Jia, C.; Yan, H., Crystal Chemistry and Magnetic Properties of Gd-Substituted Aurivillius-Type Bi5FeTi3O15 Ceramics. The Journal of Physical Chemistry C 2018, 122 (27), 15733-15743.

15. Keeney, L.; Maity, T.; Schmidt, M.; Amann, A.; Deepak, N.; Petkov, N.; Roy, S.; Pemble, M. E.; Whatmore, R. W., Magnetic Field-Induced Ferroelectric Switching in Multiferroic Aurivillius Phase Thin Films at Room Temperature. Journal of the American Ceramic Society 2013, 96 (8), 2339-2357.

16. Keeney, L.; Downing, C.; Schmidt, M.; Pemble, M. E.; Nicolosi, V.; Whatmore, R. W., Direct atomic scale determination of magnetic ion partition in a room temperature multiferroic material. Sci. Rep. 2017, 7, 1737.

17. Giddings, A.; Stennett, M.; Reid, D.; McCabe, E.; Greaves, C.; Hyatt, N., Synthesis, structure and characterization of the $\mathrm{n}=4$ Aurivillius Phase Bi5Ti3CrO15. J. Solid State Chem. 2011, 184, 252 .

18. Armstrong, R.; Newnham, R., Bismuth titanate solid solutions. Materials Research Bulletin 1972, 7 (10), 1025-1034.

19. Kikuchi, T., Stability of layered bismuth compounds in relation to the structural mismatch. Materials Research Bulletin 1979, 14 (12), 1561-1569.

20. García-Guaderrama, M.; Fuentes-Montero, L.; Rodriguez, A.; Fuentes, L., Structural characterization of Bi6Ti3Fe2O18 obtained by molten salt synthesis. Integrated ferroelectrics 2006, $83(1), 41-47$.

21. Gross, I.; Akhtar, W.; Garcia, V.; Martínez, L.; Chouaieb, S.; Garcia, K.; Carrétéro, C.; Barthélémy, A.; Appel, P.; Maletinsky, P., Real-space imaging of non-collinear antiferromagnetic order with a single-spin magnetometer. Nature 2017, 549 (7671), 252-256.

22. Hervoches, C. H.; Lightfoot, P., A variable-temperature powder neutron diffraction study of ferroelectric Bi4Ti3O12. Chemistry of materials 1999, 11 (11), 3359-3364.

23. Shrinagar, A.; Garg, A.; Prasad, R.; Auluck, S., Phase stability in ferroelectric bismuth titanate: a first-principles study. Acta Crystallographica Section A: Foundations of Crystallography 2008, 64 (3), 368-375.

24. Jia, C.-L.; Mi, S.-B.; Urban, K.; Vrejoiu, I.; Alexe, M.; Hesse, D., Atomic-scale study of electric dipoles near charged and uncharged domain walls in ferroelectric films. Nature Materials 2008, 7 (1), 57-61.

25. Borisevich, A.; Ovchinnikov, O. S.; Chang, H. J.; Oxley, M. P.; Yu, P.; Seidel, J.; Eliseev, E. A.; Morozovska, A. N.; Ramesh, R.; Pennycook, S. J.; Kalinin, S. V., Mapping Octahedral Tilts and 
Polarization Across a Domain Wall in $\mathrm{BiFeO} 3$ from Z-Contrast Scanning Transmission Electron Microscopy Image Atomic Column Shape Analysis. ACS Nano 2010, 4 (10), 6071-6079.

26. Das, S.; Tang, Y. L.; Hong, Z.; Gonçalves, M. A. P.; McCarter, M. R.; Klewe, C.; Nguyen, K. X.; Gómez-Ortiz, F.; Shafer, P.; Arenholz, E.; Stoica, V. A.; Hsu, S. L.; Wang, B.; Ophus, C.; Liu, J. F.; Nelson, C. T.; Saremi, S.; Prasad, B.; Mei, A. B.; Schlom, D. G.; Íñiguez, J.; GarcíaFernández, P.; Muller, D. A.; Chen, L. Q.; Junquera, J.; Martin, L. W.; Ramesh, R., Observation of room-temperature polar skyrmions. Nature 2019, 568 (7752), 368-372.

27. Geng, W. R.; Guo, X. W.; Zhu, Y. L.; Wang, Y. J.; Tang, Y. L.; Han, M. J.; Feng, Y. P.; Zou, M. J.; Wu, B.; Ma, J. Y.; Hu, W. T.; Ma, X. L., Oxygen octahedral coupling mediated ferroelectricantiferroelectric phase transition based on domain wall engineering. Acta Materialia 2020, 198, 145152.

28. Li, X.; Tan, C.; Liu, C.; Gao, P.; Sun, Y.; Chen, P.; Li, M.; Liao, L.; Zhu, R.; Wang, J.; Zhao, Y.; Wang, L.; Xu, Z.; Liu, K.; Zhong, X.; Wang, J.; Bai, X., Atomic-scale observations of electrical and mechanical manipulation of topological polar flux closure. Proceedings of the National Academy of Sciences 2020, 117 (32), 18954.

29. Matsumoto, T.; Ishikawa, R.; Tohei, T.; Kimura, H.; Yao, Q.; Zhao, H.; Wang, X.; Chen, D.; Cheng, Z.; Shibata, N.; Ikuhara, Y., Multivariate Statistical Characterization of Charged and Uncharged Domain Walls in Multiferroic Hexagonal YMnO3 Single Crystal Visualized by a Spherical Aberration-Corrected STEM. Nano Letters 2013, 13 (10), 4594-4601.

30. Zhang, Q.; Tan, G.; Gu, L.; Yao, Y.; Jin, C.; Wang, Y.; Duan, X.; Yu, R., Direct Observation of Multiferroic Vortex Domains in YMnO3. Scientific Reports 2013, 3 (1), 2741.

31. Holtz, M. E.; Padgett, E. S.; Steinhardt, R.; Brooks, C. M.; Meier, D.; Schlom, D. G.; Muller, D. A.; Mundy, J. A., Dimensionality-Induced Change in Topological Order in Multiferroic Oxide Superlattices. Physical Review Letters 2021, 126 (15), 157601.

32. Campanini, M.; Trassin, M.; Ederer, C.; Erni, R.; Rossell, M. D., Buried In-Plane Ferroelectric Domains in Fe-Doped Single-Crystalline Aurivillius Thin Films. ACS Applied Electronic Materials 2019, 1 (6), 1019-1028.

33. Pereira Gonçalves, M. A.; Escorihuela-Sayalero, C.; Garca-Fernández, P.; Junquera, J.; Íniguez, J., Theoretical guidelines to create and tune electric skyrmion bubbles. Science Advances 2019, 5 (2), eaau7023.

34. Mandal, P.; Pitcher, M.; Alaria, J.; Niu, H.; Borisov, P.; Stamenov, P.; Claridge, J.; Rosseinsky, M., Designing switchable polarization and magnetization at room temperature in an oxide. Nature 2015, 525 (7569), 363-366.

35. El Baggari, I.; Savitzky, B. H.; Admasu, A. S.; Kim, J.; Cheong, S.-W.; Hovden, R.; Kourkoutis, L. F., Nature and evolution of incommensurate charge order in manganites visualized with cryogenic scanning transmission electron microscopy. Proceedings of the National Academy of Sciences 2018, 115 (7), 1445.

36. Kumar, A.; Baker, J. N.; Bowes, P. C.; Cabral, M. J.; Zhang, S.; Dickey, E. C.; Irving, D. L.; LeBeau, J. M., Atomic-resolution electron microscopy of nanoscale local structure in lead-based relaxor ferroelectrics. Nature Materials 2020.

37. Mundy, J. A.; Heikes, C. A.; Grosso, B. F.; Segedin, D. F.; Wang, Z.; Goodge, B. H.; Meier, Q. N.; Nelson, C. T.; Prasad, B.; Kourkoutis, L. F., A high-energy density antiferroelectric made by interfacial electrostatic engineering. arXiv preprint arXiv:1812.09615 2018. 
38. Mundy, J. A.; Schaab, J.; Kumagai, Y.; Cano, A.; Stengel, M.; Krug, I. P.; Gottlob, D. M.; Doğanay, H.; Holtz, M. E.; Held, R.; Yan, Z.; Bourret, E.; Schneider, C. M.; Schlom, D. G.; Muller, D. A.; Ramesh, R.; Spaldin, N. A.; Meier, D., Functional electronic inversion layers at ferroelectric domain walls. Nature Materials 2017, 16 (6), 622-627.

39. Faraz, A.; Maity, T.; Schmidt, M.; Deepak, N.; Roy, S.; Pemble, M. E.; Whatmore, R. W.; Keeney, L., Direct Visualization of Magnetic-Field-Induced Magnetoelectric Switching in Multiferroic Aurivillius Phase Thin Films. J. Am. Ceram. Soc. 2017, 100 (3), 975.

40. Birenbaum, A. Y.; Ederer, C., Potentially multiferroic Aurivillius phase Bi 5 FeTi 3 O 15: cation site preference, electric polarization, and magnetic coupling from first principles. Physical Review B 2014, 90 (21), 214109.

41. Faraz, A.; Ricote, J.; Jimenez, R.; Maity, T.; Schmidt, M.; Deepak, N.; Roy, S.; Pemble, M. E.; Keeney, L., Exploring ferroelectric and magnetic properties of Tb-substituted $\mathrm{m}=5$ layered Aurivillius phase thin films. Journal of Applied Physics 2018, 123 (12), 124101.

42. Moore, K.; Conroy, M.; O’Connell, E. N.; Cochard, C.; Mackel, J.; Harvey, A.; Hooper, T. E.; Bell, A. J.; Gregg, J. M.; Bangert, U., Highly charged 180 degree head-to-head domain walls in lead titanate. Communications Physics 2020, 3 (1), 231.

43. Zhang, Y.; Tan, Y.; Sando, D.; Chen, L.-Q.; Valanoor, N.; Zhu, Y.; Han, M.-G., Controlled Nucleation and Stabilization of Ferroelectric Domain Wall Patterns in Epitaxial (110) Bismuth Ferrite Heterostructures. Advanced Functional Materials 2020, 30 (48), 2003571.

44. Daumont, C.; Ren, W.; Infante, I.; Lisenkov, S.; Allibe, J.; Carrétéro, C.; Fusil, S.; Jacquet, E.; Bouvet, T.; Bouamrane, F., Strain dependence of polarization and piezoelectric response in epitaxial BiFeO3 thin films. Journal of Physics: Condensed Matter 2012, 24 (16), 162202.

45. Bednyakov, P. S.; Sturman, B. I.; Sluka, T.; Tagantsev, A. K.; Yudin, P. V., npj Comput. Mater. 2018, 4, 65.

46. Liu, Y.; Zhu, Y.-L.; Tang, Y.-L.; Wang, Y.-J.; Li, S.; Zhang, S.-R.; Han, M.-J.; Ma, J.-Y.; Suriyaprakash, J.; Ma, X.-L., Controlled growth and atomic-scale mapping of charged heterointerfaces in $\mathrm{PbTiO} 3 / \mathrm{BiFeO} 3$ bilayers. ACS applied materials \& interfaces 2017, 9 (30), 25578-25586.

47. Scott, J., Multiferroic memories. Nature materials 2007, 6 (4), 256-257.

48. Yin, Y.-W.; Raju, M.; Hu, W.-J.; Weng, X.-J.; Zou, K.; Zhu, J.; Li, X.-G.; Zhang, Z.-D.; Li, Q., Multiferroic tunnel junctions. Frontiers of Physics 2012, 7 (4), 380-385.

49. Rojac, T.; Bencan, A.; Drazic, G.; Sakamoto, N.; Ursic, H.; Jancar, B.; Tavcar, G.;

Makarovic, M.; Walker, J.; Malic, B., Domain-wall conduction in ferroelectric BiFeO 3 controlled by accumulation of charged defects. Nature materials 2017, 16 (3), 322-327.

50. Catalan, G.; Seidel, J.; Ramesh, R.; Scott, J. F., Domain wall nanoelectronics. Reviews of Modern Physics 2012, 84 (1), 119-156.

51. Seidel, J., Topological structures in ferroic materials. Switzerland: Springer International Publishing 2016.

52. Zubko, P.; Wojdeł, J. C.; Hadjimichael, M.; Fernandez-Pena, S.; Sené, A.; Luk'yanchuk, I.; Triscone, J.-M.; Íñiguez, J., Negative capacitance in multidomain ferroelectric superlattices. Nature 2016, 534 (7608), 524-528.

53. Wojdeł, J. C.; Íñiguez, J., Ab initio indications for giant magnetoelectric effects driven by structural softness. Physical review letters 2010, 105 (3), 037208. 
54. Birenbaum, A. Y.; Ederer, C., Potentially multiferroic Aurivillius phase Bi5FeTi3O15: Cation site preference, electric polarization, and magnetic coupling from first principles. Phys. Rev. B: Condens. Matter Mater. Phys. 2014, 90, 214109.

55. Huang, R.; Ding, H. C.; Liang, W. I.; Gao, Y. C.; Tang, X. D.; He, Q.; Duan, C. G.; Zhu, Z.; $\mathrm{Chu}$, J.; Fisher, C. A., Atomic-scale visualization of polarization pinning and relaxation at coherent BiFeO3/LaAlO3 interfaces. Advanced Functional Materials 2014, 24 (6), 793-799.

56. Béa, H.; Dupé, B.; Fusil, S.; Mattana, R.; Jacquet, E.; Warot-Fonrose, B.; Wilhelm, F.; Rogalev, A.; Petit, S.; Cros, V., Evidence for room-temperature multiferroicity in a compound with a giant axial ratio. Physical review letters 2009, 102 (21), 217603.

57. Moure, A., Review and perspectives of aurivillius structures as a lead-free piezoelectric system. Applied Sciences 2018, 8 (1), 62.

58. Pignolet, A.; Schäfer, C.; Satyalakshmi, K. M.; Harnagea, C.; Hesse, D.; Gösele, U., Orientation dependence of ferroelectricity in pulsed-laser-deposited epitaxial bismuth-layered perovskite thin films. Applied Physics A 2000, 70 (3), 283-291.

59. Newnham, R. E.; Wolfe, R. W.; Dorrian, J. F., Structural basis of ferroelectricity in the bismuth titanate family. Materials Research Bulletin 1971, 6 (10), 1029-1039.

60. Aschauer, U.; Pfenninger, R.; Selbach, S. M.; Grande, T.; Spaldin, N. A., Strain-controlled oxygen vacancy formation and ordering in $\mathrm{CaMnO} \$\{\} \_\{3\}$ \$. Physical Review B 2013, 88 (5), 054111 .

61. Farokhipoor, S.; Noheda, B., Local conductivity and the role of vacancies around twin walls of (001)-BiFeO3 thin films. Journal of Applied Physics 2012, 112 (5), 052003.

62. Du, K.; Zhang, M.; Dai, C.; Zhou, Z. N.; Xie, Y. W.; Ren, Z. H.; Tian, H.; Chen, L. Q.; Van Tendeloo, G.; Zhang, Z., Manipulating topological transformations of polar structures through realtime observation of the dynamic polarization evolution. Nature Communications 2019, 10 (1), 4864. 63. Zurbuchen, M.; Tian, W.; Pan, X.; Fong, D.; Streiffer, S.; Hawley, M.; Lettieri, J.; Jia, Y.; Asayama, G.; Fulk, S., Morphology, structure, and nucleation of out-of-phase boundaries (OPBs) in epitaxial films of layered oxides. Journal of materials research 2007, 22 (6), 1439-1471.

64. Gregg, J., Exotic domain states in ferroelectrics: searching for vortices and skyrmions. Ferroelectrics 2012, 433 (1), 74-87.

65. Yadav, A.; Nelson, C.; Hsu, S.; Hong, Z.; Clarkson, J.; Schlepütz, C.; Damodaran, A.; Shafer, P.; Arenholz, E.; Dedon, L., Observation of polar vortices in oxide superlattices. Nature 2016, 530 (7589), 198-201.

66. Damodaran, A. R.; Clarkson, J.; Hong, Z.; Liu, H.; Yadav, A. K.; Nelson, C. T.; Hsu, S.-L.; McCarter, M.; Park, K.-D.; Kravtsov, V., Phase coexistence and electric-field control of toroidal order in oxide superlattices. Nature materials 2017, 16 (10), 1003-1009.

67. Nelson, C. T.; Winchester, B.; Zhang, Y.; Kim, S. J.; Melville, A.; Adamo, C.; Folkman, C. M.; Baek, S. H.; Eom, C. B.; Schlom, D. G.; Chen, L. Q.; Pan, X., Spontaneous Vortex Nanodomain Arrays at Ferroelectric Heterointerfaces. Nano Lett. 2011, 11, 828.

68. Li, Y.; Jin, Y. M.; Lu, X. M.; Yang, J. C.; Chu, Y. H.; Huang, F. Z.; Zhu, J. S.; Cheong, S. W., Rewritable Ferroelectric Vortex Pairs in BiFeO3. npj Quantum Mater. 2017, 2, 43.

69. Hong, Z.; Das, S.; Nelson, C.; Yadav, A.; Wu, Y.; Junquera, J.; Chen, L.-Q.; Martin, L. W.; Ramesh, R., Vortex Domain Walls in Ferroelectrics. Nano Letters 2021, 21 (8), 3533-3539.

70. Martin, L. W., Whirls and swirls of polarization. Science 2021, 371 (6533), 992-993. 
71. Houchmandzadeh, B.; Lajzerowicz, J.; Salje, E., Order parameter coupling and chirality of domain walls. Journal of Physics: Condensed Matter 1991, 3 (27), 5163-5169.

72. Daraktchiev, M.; Catalan, G.; Scott, J. F., Landau theory of domain wall magnetoelectricity. Physical Review B 2010, 81 (22), 224118.

73. Song, D.; Yang, J.; Yang, B.; Chen, L.; Wang, F.; Zhu, X., Evolution of structure and ferroelectricity in Aurivillius Bi $4 \mathrm{Bi} \mathrm{n}-3 \mathrm{Fe} \mathrm{n}-3$ Ti 3 O 3n+ 3 thin films. Journal of Materials Chemistry C 2018, 6 (32), 8618-8627.

74. Balke, N.; Winchester, B.; Ren, W.; Chu, Y. H.; Morozovska, A. N.; Eliseev, E. A.; Huijben, M.; Vasudevan, R. K.; Maksymovych, P.; Britson, J.; Jesse, S.; Kornev, I.; Ramesh, R.; Bellaiche, L.; Chen, L. Q.; Kalinin, S. V., Enhanced electric conductivity at ferroelectric vortex cores in BiFeO3. Nature Physics 2012, 8 (1), 81-88.

75. Hundley, M.; Neumeier, J., Thermoelectric power of La 1-x $\mathrm{Ca} \times \mathrm{MnO} 3+\delta$ : Inadequacy of the nominal Mn 3+/4+ valence approach. Physical Review B 1997, 55 (17), 11511.

76. Nord, M.; Vullum, P. E.; MacLaren, I.; Tybell, T.; Holmestad, R., Atomap: a new software tool for the automated analysis of atomic resolution images using two-dimensional Gaussian fitting. Advanced Structural and Chemical Imaging 2017, 3 (1), 9.

77. Eoghan O'Connell, M. H., Eoin Moynihan PinkShnack/TEMUL: Version 0.1.3 (0.1.3). Zenodo.

78. Grillo, V.; Rotunno, E., STEM_CELL A Software Tool for Electron Microscopy Part ISimulations. Ultramicroscopy 2013, 125 (C), 97.

79. Kresse, G.; Hafner, J., Ab initio molecular-dynamics simulation of the liquid-metal-amorphous-semiconductor transition in germanium. Physical Review B 1994, 49 (20), 14251-14269.

80. Kresse, G.; Furthmuller, J., Efficient Iterative Schemes for ab Initio Total-Energy

Calculations Using a Plane-Wave Basis Set. Phys. Rev. B 1996, 54, 11169.

81. Blöchl, P. E., Projector Augmented-Wave Method. Phys. Rev. B: Condens. Matter Mater. Phys. 1994, 50, 17953.

82. Perdew, J. P.; Burke, K.; Ernzerhof, M., Generalized Gradient Approximation Made Simple. Phys. Rev. Lett. 1996, 77, 3865.

83. Weber, S. F.; Griffin, S. M.; Neaton, J. B., Topological semimetal features in the multiferroic hexagonal manganites. Physical Review Materials 2019, 3 (6), 064206.

84. Dudarev, S. L.; Botton, G. A.; Savrasov, S. Y.; Humphreys, C. J.; Sutton, A. P., Electronenergy-loss spectra and the structural stability of nickel oxide: An LSDA+U study. Physical Review B 1998, 57 (3), 1505-1509. 


\section{Supplementary Files}

This is a list of supplementary files associated with this preprint. Click to download.

- SIChargedDomainWallandPolarVortexTopologiesinaRoomTemperatureMagnetoelectricMultiferroicThinFilm.pdf 